\title{
Nutrition lipidique, inflammation et tissu osseux
}

\author{
Fabien WAUQUIER \\ Véronique COXAM \\ Yohann WITTRANT \\ Inra, UMR 1019, UNH, CRNH \\ Auvergne, F-63009 Clermont-Ferrand ; \\ Clermont Université, Université \\ d'Auvergne, Unité de Nutrition \\ Humaine, BP 10448, F-63000 \\ Clermont-Ferrand ; Équipe Alimentation, \\ squelette et métabolismes \\ $<$ yohann.wittrant@clermont.inra.fr>
}

\section{Contexte}

Tous les pays industrialisés font face à une augmentation progressive de l'espérance de vie en raison du recul de la mortalité. Par conséquent, la prévalence des maladies chroniques liées à l'âge a augmenté. Ces complications métaboliques sont associées à des dysfonctionnements de l'appareil locomoteur. Parmi ces maladies, la sarcopénie et l'ostéoporose sont particulièrement invalidantes et représentent un enjeu social et économique à travers le monde. En ce qui concerne la qualité de vie, I'ostéoporose a des effets dévastateurs psychologiques et socio-économiques. Les fractures ostéoporotiques peuvent augmenter jusqu'à $20 \%$ le taux de mortalité dans I'année qui suit la fracture. En outre, jusqu'à $50 \%$ des patients ne récupèreront pas la totalité de leurs facultés physiques, la moitié d'entre eux nécessitant des soins de longue durée à domicile. Seulement un tiers se remettra complètement du traumatisme.

L'ostéoporose est caractérisée par une fragilité osseuse excessive qui conduit à un risque accru de fractures. Les traitements d'aujourd'hui tels que le

\begin{abstract}
Fats are prevalent in western diets; they have known deleterious effects on muscle insulin resistance and may contribute to bone loss most notably in the elderly population. Because current treatments for osteoporosis may lead to important side effects, several studies aimed at investigating the relevance of nutritional approaches and most notably the role of lipid diets on bone health status. Literature has widely linked lipid intake and inflammation status, a key protagonist involved in bone resorption. Regarding inflammation, lipids exhibit a duality, with both pro- and anti-inflammatory effects depending on their structures and metabolism. In this light, a growing body of evidence has revealed that $\varpi-6$ increase bone loss while $\varpi-3$ are believed to protect bone health. Nevertheless, this debate remains controversial and the mechanisms of action are poorly understood.
\end{abstract}

Key words: bone, inflammation, fatty acids, osteoporosis, ageing

traitement hormonal substitutif (THS) ont démontré des avantages évidents sur les femmes ostéoporotiques postménopausées (Ozgocmen et al., 2007; Mann et al., 2007). Toutefois, une prévalence plus élevée d'accidents thrombo-emboliques, de cancers de I'utérus et du sein a été signalée (Valverde, 2008). Parce que la recherche en nutrition au cours des trente dernières années a conduit à soutenir I'hypothèse qu'en modulant les fonctions cibles spécifiques de l'organisme, I'alimentation peut aider à atteindre une santé optimale en réduisant le risque de maladie, plusieurs études ont été consacrées à l'investigation du rôle de I'alimentation lipidique sur notre santé osseuse.

\section{Le tissu osseux}

Le squelette représente la charpente de l'organisme. À l'âge adulte, l'individu possède un tissu osseux minéralisé sur lequel seules subsistent des zones de cartilage articulaire. La minéralisation confère à l'os des propriétés particulières, notamment mécaniques et métaboliques. L'os minéralisé est rigide et permet le maintient de la stature de l'organisme. Outre la station debout, le squelette humain assure une fonction de protection des organes vitaux. Ceci est particulièrement vérifiable pour le système nerveux central, le cœur et les poumons protégés par les os du crâne et la cage thoracique, respectivement. Le tissu osseux intervient également dans le déplacement de l'individu et constitue un élément central de l'appareil locomoteur. En outre, le tissu osseux renferme la moelle osseuse, siège de l'hématopoï̀se. D'autre part, par sa composition le tissu minéralisé participe à la régulation de la calcémie.

L'os est le siège d'activités de résorption et d'apposition coordonnées dans le temps correspondant au remodelage osseux. Ce processus est le fait de deux types cellulaires, les ostéoclastes et les ostéoblastes qui en réponse à différents stimuli assurent la croissance, le renouvellement du tissu osseux et le maintient de I'homéostasie phospho-calcique. Les activités ostéoblastique et ostéoclastique sont respectivement responsables des phénomènes d'apposition et de résorption au sein du tissu osseux. L'équilibre entre ces phases permet à

Pour citer cet article : Wauquier F, Coxam V, Wittrant Y. Nutrition lipidique, inflammation et tissu osseux. OCL 2011; 18(1) : 31-33. doi : 10.1684/ocl.2011.0365 
I'os de conserver ses propriétés. Cet équilibre est finement régulé par l'action de nombreux facteurs locaux et systémiques tels que des cytokines, des hormones mais aussi par des interactions cellulaires. Parmi les acteurs principaux, la triade de molécules récemment découvertes: Receptor Activator of NFkB/RANK Ligand/Ostéoprotégérine (RANK/RANKL/OPG) représente un pivot moléculaire vers lequel convergent la plupart des systèmes régulateurs de la résorption osseuse connus à ce jour (Theoleyre et al., 2004).

\section{Lipides et tissu osseux}

Les effets de la nutrition lipidique sur le tissu osseux doivent être considérés tant sur le plan quantitatif que qualitatif. Des méta-anlyses récentes montrent que malgré la stimulation mécanique de l'os lors de l'établissement de l'obésité, les régimes hyperlipidiques ont un effet délétère sur la matrice osseuse. Sur le plan qualitatif, des études cliniques et précliniques démontrent que l'effet sur le tissu osseux varie en fonction du degré de saturation et de la longueur des chaines $\mathrm{d}^{\prime}$ acides gras. Les modalités d'action des acides gras sont plurielles. Leurs effets peuvent être médiés via un récepteur nucléaire ou membranaire, une modification de la fluidité membranaire, de la synthèse de dérivés lipidiques (éicosanoïdes, docosanoïdes, céramides), une modulation de l'expression de facteurs de l'inflammation, d'adipokines ou encore du profil adipeux de la moelle osseuse.

Le paramètre le plus décrit est une modulation du contexte inflammatoire. En effet, la littérature a largement liée I'apport lipidique et le statut inflammatoire, un protagoniste clé impliqué dans la résorption osseuse (Ono et al., 2005; Tsutsumi et al., 2009). En effet, l'inflammation favorise la dégradation osseuse en stimulant l'activité des ostéoclastes, tout en inhibant la formation osseuse ostéoblastique entraînant un déséquilibre du remodelage osseux et une perte osseuse.

À ce sujet, les lipides présentent une dualité d'action, avec tantôt des effets pro- et tantôt des effets anti-inflammatoires en fonction de leurs structures et de leur métabolisme (Laneuville et al., 1995; Raisz et al., 1989). Dans ce contexte, un nombre croissant de publications a permis de mettre en évidence que les acides gras de la famille des $\varpi-6$ étaient associés à une perte osseuse accrue, tandis que les $\varpi$ - 3 sont pressentis pour protéger le capital osseux (Ono et al., 2005; Tsutsumi et al., 2009; Corwin, 2003; Calder, 2006). Néanmoins, ce débat reste controversé et les mécanismes d'action sont mal connus.

\section{Principaux acteurs et principales cibles}

La prostaglandine E2 est un dérivé de I'acide arachidonique ( $\bar{\omega}-6)$. Produite par cyclo-oxygénation, elle est la prostaglandine la plus étudiée au niveau osseux en raison de sa contribution majeure à l'établissement d'un contexte proinflammatoire local qui favorise la résorption et la perte osseuse. Sa synthèse peut être ralentie lors d'un régime enrichi en $\varpi$ - 3 ou en certains $\varpi$ - 6 tels que l'acide $\gamma$-linolénique, démontrant ainsi le rôle complexe que peut avoir la nutrition lipidique au sein des mécanismes de remodelage osseux.

Dans la compétition que se livrent les différentes classes d'acides gras, la notion de cible cellulaire est à prendre en compte. En effet, les acides gras impactent à la fois l'activité des ostéoblastes, des ostéoclastes ainsi que leur couplage. Plusieurs équipes ont pu mettre en évidence une corrélation inverse entre les ratios RANKL/OPG et $\varpi-3 /$ / 6 (Poulsen et al., 2008). Concernant les ostéoblastes, la translocation de runx2, un facteur de transcription majeur dans la différentiation ostéoblastique, est augmentée en présence de $\varpi$-3 (Shen et al., 2006). Ces données sont corrélées à la diminution de production de cytokines de l'inflammation telles que I'IL-6 et le TNF $\alpha$ par les ostéoblastes en présence $d^{\prime}$ acides gras de la famille des ๗-3 et supportent le rôle bénéfique de cette classe sur les paramètres osseux.

En parallèle, alors que la PGE2 augmente la taille, I'activité et la viabilité des ostéoclastes, l'ostéoclastogenèse est inhibée par le DHA (ळ-3), notamment via une diminution de la synthèse et de l'activité du facteur de transcription NFkB (Ono et al., 2005; Tsutsumi et al., 2009; Rahman et al., 2008) un acteur moléculaire clé dans les voies de différentiation ostéoclastique et de synthèse de cytokines pro-inflammatoires.

\section{Nutrition lipidique et ostéoporose}

Notre équipe vient de terminer une étude préclinique dont le but était d'analyser l'impact de la qualité des acides gras sur l'évolution de l'appareil locomoteur liée à l'âge afin de mieux comprendre quels mécanismes de vieillissement sont impliqués. La souche de souris SAMP8 a été choisie comme modèle de progeria, en comparaison de la souche témoin SAMR1. À deux mois, les souris ont été divisées en différents groupes et soumises aux régimes suivants :

1) "standard de croissance "

2) “tournesol " (ratio élevé $\varpi 6 / \varpi 3$ )

3 ) " bourrache » (haute teneur en acide $\gamma$-linolénique)

4) "poisson" (riche en $\varpi 3$ à longue chaîne).

Les souris ont été nourries ad libitum pendant l'ensemble du protocole. À 12 mois, les souris ont été sacrifiées et les tissus ont été prélevés pour des études d'architecture osseuse, de mesure de la masse grasse et musculaire, des paramètres de l'inflammation et de l'expression des marqueurs des cellules osseuses. Après validation de l'établissement d'un tableau clinique I'ostéoporotique sénile dans notre modèle, nous avons pu démontrer un effet préventif des régimes de bourrache et de poisson sur l'évolution des paramètres de l'inflammation et des paramètres osseux. Ces données viennent supporter la pertinence d'une approche nutritionnelle pour l'établissement de stratégies de prévention des dysfonctionnements locomoteurs liés à l'âge.

\section{RÉFÉRENCES}

Calder PC. n-3 polyunsaturated fatty acids, inflammation, and inflammatory diseases. Am J Clin Nutr 2006 ; 83 : 1505S-19S.

Corwin RL. Effects of dietary fats on bone health in advanced age. Prostaglandins leukotrienes and essential fatty acids 2003 ; 68 : 379-86.

Laneuville O, Breuer DK, Xu N, et al. Fatty acid substrate specificities of human prostaglandin-endoperoxide $\mathrm{H}$ synthase-1 and -2. Formation of 12-hydroxy-(9Z, 13E/Z, 15Z)octadecatrienoic acids from alpha-linolenic acid. The J Biol Chem 1995 ; 270 : 19330-6.

Mann V, Huber C, Kogianni G, Collins F, Noble B. The antioxidant effect of estrogen 
and Selective Estrogen Receptor Modulators in the inhibition of osteocyte apoptosis in vitro. Bone 2007 ; 40 : 674-84.

Ono K, Kaneko $\mathrm{H}$, Choudhary $\mathrm{S}$, et al. Biphasic effect of prostaglandin E2 on osteoclast formation in spleen cell cultures: role of the EP2 receptor. / Bone Miner Res $2005 ; 20: 23-9$.

Ozgocmen S, Kaya H, Fadillioglu E, Aydogan R, Yilmaz Z. Role of antioxidant systems, lipid peroxidation, and nitric oxide in postmenopausal osteoporosis. Mol Cell Biochem 2007 ; 295 : 45-52.

Poulsen RC, Wolber FM, Moughan PJ, Kruger MC. Long chain polyunsaturated fatty acids alter membrane-bound RANK-L expression and osteoprotegerin secretion by MC3T3-E1 osteoblast-like cells. Prostaglandins \& Other Lipid Mediators 2008 ; 85 : 42-8.

Rahman MM, Bhattacharya A, Fernandes G. Docosahexaenoic acid is more potent inhibitor of osteoclast differentiation in RAW 264.7 cells than eicosapentaenoic acid. J Cell Physiol 2008 ; 214 : 201-9.

Raisz LG, Alander CB, Simmons HA. Effects of prostaglandin E3 and eicosapentaenoic acid on rat bone in organ culture. Prostaglandins $1989 ; 37: 615-25$.

Shen CL, Yeh JK, Rasty J, Li Y, Watkins BA. Protective effect of dietary long-chain $n-3$ polyunsaturated fatty acids on bone loss in gonad-intact middle-aged male rats. $\mathrm{Br}$ J Nutr 2006 ; $95: 462-8$.

Theoleyre S, Wittrant Y, Tat SK, Fortun Y, Redini F, Heymann D. The molecular triad OPG/RANK/RANKL: involvement in the orchestration of pathophysiological bone remodeling. Cytokine \& Growth Factor Reviews $2004 ; 15$ : 457-75.

Tsutsumi R, Xie C, Wei X, et al. PGE2 signaling through the EP4 receptor on fibroblasts upregulates RANKL and stimulates osteolysis. J Bone Miner Res 2009 ; 24 : 1753-62.

Valverde P. Pharmacotherapies to manage bone loss-associated diseases: a quest for the perfect benefit-to-risk ratio. Curr Med Chem 2008 ; 15 : 284-304. 